\title{
Detection of antibodies to the $\alpha 4 \beta 7$ integrin binding site on HIV-1 gp120 V2 loop using a novel cell adhesion assay
}

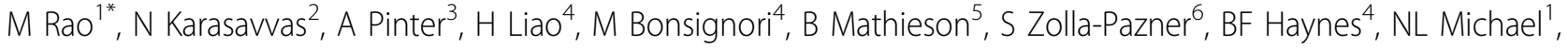 \\ JH Kim¹, CR Alving ${ }^{1}$, KK Peachman ${ }^{7}$
}

From AIDS Vaccine 2012

Boston, MA, USA. 9-12 September 2012

\section{Background}

The gut mucosal homing integrin receptor $\alpha 4 \beta 7$ present on activated CD4+ T-cells interacts with the HIV-1 gp120 second variable loop (V2). Case control analysis of the RV144 phase III vaccine trial showed that antibodies induced by the vaccine bound to a MuLV-gp70 scaffolded V1V2 loop of gp120 (V1V2-gp70) and correlated inversely with infection. These, and other data, generate the hypothesis that the vaccine-elicited antibodies may have been involved in limiting HIV-1 acquisition. We have developed a high-throughput assay to evaluate antibodies that block $\alpha 4 \beta 7$ binding. We have named this the RAP assay.

\section{Methods}

Plates were coated with either MAdCAM-1, the natural ligand of $\alpha 4 \beta 7$, or streptavidin followed by the addition of biotinylated cyclic-V2 peptides (strain $92 \mathrm{TH} 023$ or MN). Plasma and purified IgG antibodies from RV144 volunteers, conformational mAbs specific for V2 (697), for V2 and V3 (PG9, PG16), or for V2 linear epitopes (CH58 and $\mathrm{CH} 59$, cloned from RV144 vaccinee B cells) were then added to the peptide-coated plates followed by RPMI8866 cells, which constitutively express $\alpha 4 \beta 7$. Cell binding/inhibition was assessed by AlamarBlue. Anti- $\alpha 4 \beta 7$-specific $\mathrm{mAb}$ (ACT-1) served as a control. In separate experiments, plasma, IgG, or mAbs were tested in a competition assay using V1V2-gp70.

\section{Results}

ACT-1 inhibited the binding of both MAdCAM-1 and cyclic-V2 peptides to $\alpha 4 \beta 7$ by $65-85 \%$, while $\mathrm{CH} 58$ and
$\mathrm{CH} 59$ inhibited the $\alpha 4 \beta 7$-cyclic-V2 peptide binding by $37-45 \%$ in a dose-dependent manner. PG9, PG16, and 697 did not inhibit the binding of V2-peptides to the cells. However, in the competition assay, 697 and PG9 mAbs inhibited V1V2-gp70 binding to $\alpha 4 \beta 7$. Some of the RV144 plasma and IgG inhibited binding to 92TH023 or MN-V2-peptides.

\section{Conclusion}

We have developed a novel high-throughput reproducible assay for assessing $\alpha 4 \beta 7$-specific blocking antibodies. The above results raise the hypothesis that anti-V2 loop antibodies may play a role in regulation of gp120- $\alpha 4 \beta 7$ interaction.

\section{Author details}

${ }^{1}$ U.S. Military HIV Research Program, WRAIR, Silver Spring, MD, USA. ${ }^{2}$ Armed Forces Research Institute of Medical Science (AFRIMS), Bangkok, Thailand. ${ }^{3}$ The Public Health Research Institute Center at the International Cente, Newark, NJ, USA. ${ }^{4}$ Duke Human Vaccine Institute Duke University Medical Center, Durham, NC, USA. ${ }^{5}$ Office of AIDS Research, National Institutes of Health, Bethesda, MD, USA. ${ }^{6} \mathrm{NYU}$ School of Medicine, New York, NY, USA. ${ }^{7}$ United States Military HIV Research Program, WRAIR / HJF, Silver Spring, MD, USA.

Published: 13 September 2012

doi:10.1186/1742-4690-9-S2-P71

Cite this article as: Rao et al.: Detection of antibodies to the $\alpha 4 \beta 7$ integrin binding site on HIV-1 gp120 V2 loop using a novel cell adhesion assay. Retrovirology 2012 9(Suppl 2):P71. 